\title{
Belief Change in Nonmonotonic Multi-Context Systems
}

\author{
Yisong Wang ${ }^{1}$, Zhiqiang Zhuang ${ }^{2}$, and Kewen Wang ${ }^{2}$ \\ 1 Department of Computer Science, Guizhou University, Guiyang, 550025, China \\ 2 School of Information and Communication Technology, Griffith University, Australia
}

\begin{abstract}
Brewka and Eiter's nonmonotonic multi-context systems is an elegant knowledge representation framework to model heterogeneous and nonmonotonic multiple contexts. Belief change is a central problem in knowledge representation and reasoning. In the paper we follow the classical AGM approach to investigate belief change in multi-context systems. Specifically, we semantically formulate the AGM postulates of belief expansion, revision and contraction for multi-context systems. We show that the belief revision can be characterized in terms of minimal change by ordering equilibria of multi-context systems. Two distance based revision operators are obtained and related to the classical Satoh and Dalal revision operators (via loop formulas).
\end{abstract}

\section{Introduction}

Knowledge Representation and Reasoning (KR) is a long-standing and traditional research area, which plays a crucial role in artificial intelligence and computer science. A mass of logical theories have been proposed for KR, including monotonic and nonmonotonic ones [16]. Among the latter, logic programming based on answer sets semantics (ASP) is distinguished due to its elegant theoretical foundation and existing efficient implementations for various applications $[15,5]$.

Over the last decades, there has been increasing interest in KR systems comprising multiple knowledge bases. It leads to the development of Multi-Context Systems (MCS), which builds up on several theories (the contexts) that are interlinked with bridge rules so that it allows to incorporate knowledge into a context according to knowledge in other contexts [26,3]. The abstract and general MCS framework of Brewka and Eiter [3] is of special interest since it allows for heterogeneous and nonmonotonic MCS in two aspects. On the one hand, every context may have different and nonmonotonic logics; on the other hand, bridge rules may use default negation. It raises many attractive applications in interesting scenarios [4].

As knowledge is continually evolving and subject to change, change of logical theories and knowledge base is a central issue in KR. Three major operations on belief change are expansion, revision and contraction [23]. Among the work on belief change, the most influential approach is AGM model, where a set of postulates is proposed for rational belief change operations [1]. Those postulates for propositional knowledge bases are semantically characterized in terms of minimal change [17].

In nonmonotonic logics and ASP in particular, the problem of knowledge base change appears to be intrinsically much more difficult than in a monotonic setting. For answer set programming, there has been substantial efforts in developing belief 
change, several approaches have been proposed, and some of them are under the title update $[27,2,12,24,9]$. As multi-context systems may refer to multiple individual (nonmonotonic) contexts, belief change in MCS is highly possible and intricate because of nonmonotonic bridge rules, thus a challenge. Let us consider the following scenario.

Example 1. [A running example] John went to hospital for a high fever lasting more than a week. Doctors Smith and Alice were assigned to diagnose John's high fever in two rounds. In the first round of diagnosis, while Smith believes that there is no bird flu, Alice thinks that it is due to either bird flue or pneumonia, and she believes that pneumonia means no swine flu. They both agree that if one concludes with bird/swine flu then the other does.

In the second round of diagnosis, while Alice has the same knowledge as in the first round, Smith believes that John got either bird flu or swine flu. And the agreement between Smith and Alice are same as in first round. In terms of the second round of diagnosis, how should we update/change the beliefs about John's high fever in the first round of diagnosis?

In this paper we follow the classical AGM approach to address the above belief change issue in multi-context systems. We formulate the postulates of belief change (expansion, revision and contraction) for multi-context systems in a semantic manner. By an ordering on equilibria of multi-context systems, we characterize the postulates of belief revision operators in terms of minimal change. According to the generalized Satoh and Dalal distance [25,6] among equilibria, two specific revision operators for multi-context systems are obtained. Since the equilibria of a multi-context system can be captured by the models of a corresponding propositional theory, i.e. loop formulas [7], we establish a connection between the revisions for multi-context systems and propositional theories.

The rest of the paper is organized as follows. In the next section, we briefly recall the basic notions of AGM model for belief change and multi-context systems. In Section 3, we formulate the belief change postulates for expansion, revision and contraction. Their properties are investigated. Related work and future work are discussed in Section 4. Due to space constraints, the proofs for theorems are sketched.

\section{Preliminaries}

In the section we briefly recall basic notions of belief change $[1,17]$ and multi-context systems [3,4]. For a comprehensive overview of AGM model, see [13].

\subsection{Classical belief revision}

The three major belief changes are expansion, revision and contraction. While expansion means addition of a belief without checking inconsistency, contraction means removal of a belief, revision stands for addition of a belief while maintaining consistency.

The most recognized belief change theory is AGM model [1]. Katsuno and Mendelzon semantically formulated AGM belief revision postulates as an operator $\dot{+}: L \times L \rightarrow$ $L$ satisfying the following conditions [17]:

(R1) $\alpha \dot{+} \beta \vdash \beta$. 
(R2) If $\alpha \wedge \beta$ is satisfiable then $\alpha \dot{+} \beta \equiv \alpha \wedge \beta$.

(R3) If $\beta$ is satisfiable then $\alpha+\beta$ is also satisfiable.

(R4) If $\alpha_{1} \equiv \alpha_{2}$ and $\beta_{1} \equiv \beta_{2}$ then $\alpha_{1} \dot{+} \beta_{1} \equiv \alpha_{2} \dot{+} \beta_{2}$.

(R5) $(\alpha \dot{+} \beta) \wedge \gamma \vdash \alpha \dot{+}(\beta \wedge \gamma)$.

(R6) If $(\alpha \dot{+} \beta) \wedge \gamma$ is satisfiable then $\alpha \dot{+}(\beta \wedge \gamma) \vdash(\alpha \dot{+} \beta) \wedge \gamma$.

Here we assume that $L$ is a propositional language over a finite signature and we fix a way of representing any deductively closed set $K$ of formulas by a propositional formula $\psi$ such that $K=\{\phi \mid \psi \vdash \phi\}$. The intuition behind the first four postulates can be easily read out, e.g., the new knowledge $\beta$ is kept in the updated knowledge base by (R1). The last two postulates express that the revision by a conjunction is the same as revision by one conjunct conjoined with the other conjunct if the result is satisfiable.

In proportional logic, the belief expansion is quite natural and trivial which is defined as $\alpha+\beta={ }_{\text {def }} C n(\alpha \cup \beta)$, i.e. $\{\phi \mid \alpha \wedge \beta \vdash \phi\}$. The revision and contraction ( $\left.\dot{-}\right)$ can be defined by each other via identities and expansion as follows:

- Levi identity: $\alpha \dot{+} \beta={ }_{\text {def }}(\alpha \dot{-} \neg \beta)+\beta$,

- Harper identity: $\alpha \dot{-} \beta={ }_{\text {def }} \alpha \cap(\alpha \dot{+} \neg \beta)$.

Recall that formulas are identified as deductively closed sets (called knowledge sets).

\subsection{Nonmonotonic multi-context systems}

An (abstract) logic $L$ is a tuple $L=\left(\mathbf{K B}_{L}, \mathbf{B} \mathbf{S}_{L}, \mathbf{A} \mathbf{C C}_{L}\right)$, where

- $\mathbf{K B}_{L}$ is a set of well-formed knowledge bases, each being a set (of formulas),

- $\mathbf{B S}_{L}$ is a set of possible belief sets, each being a set (of formulas),

- $\mathbf{A C C}_{L}: \mathbf{K B}_{L} \rightarrow 2^{\mathbf{B S}_{L}}$ assigns to each $k b \in \mathbf{K B}_{L}$ a set of acceptable belief sets.

For our belief change purpose, we assume that (i) the signature of $L$ is finite, (ii) each belief set of $L$ can be represented as a set of atoms containing no free variables and, (iii) for every collection $\mathbb{M}_{L}$ of belief sets of $L$ there exists a knowledge base $k b$ such that $\mathbf{A C C}_{L}(k b)=\mathbb{M}_{L}$. The ASP logic defined in [14] and propositional logic satisfy the conditions (ii) and (iii). Note that if $L$ is a propositional logic over finite signature then every formula $\alpha$ of $L$ can be identified with its deductive closure, i.e. $\{\phi \mid \alpha \vdash \phi\}$. In the following we identify a formula (and a single element set of formulas) $\alpha$ with $C n(\alpha)$ when it is clear from context.

Given a sequence $\mathcal{L}$ of abstract logics $\left(L_{1}, \ldots, L_{n}\right)$, an indexed atom of $\mathcal{L}$ is an expression of the form $(i: p)$ where $1 \leq i \leq n$ and $p$ is an element of some belief sets of $L_{i}$. An $L_{i}$-bridge rule $r$ over $\mathcal{L}$ is an expression of the form

$$
p \leftarrow\left(c_{1}: p_{1}\right), \ldots,\left(c_{m}: p_{m}\right), \operatorname{not}\left(c_{m+1}: p_{m+1}\right), \ldots, \operatorname{not}\left(c_{k}: p_{k}\right)
$$

where $p$ is a formula of $L_{i}=\left(\mathbf{K B}_{i}, \mathbf{B S}_{i}, \mathbf{A C C}_{i}\right)$ such that $\{p\} \cup k b_{i} \in \mathbf{K B}_{i}$ for any $k b_{i} \in \mathbf{K B}_{i}$, and each $\left(c_{i}: p_{i}\right)$ is an indexed atom of $\mathcal{L}$. We denote $\operatorname{Head}(r)=p$, $\operatorname{Pos}(r)=\left\{\left(c_{i}: p_{i}\right) \mid 1 \leq i \leq m\right\}$ and $\operatorname{Neg}(r)=\left\{\left(c_{j}: p_{j}\right) \mid m+1 \leq j \leq k\right\}$.

Definition 1. A multi-context system (MCS in short) $\alpha=\left(C_{1}, \ldots, C_{n}\right)$ consists of contexts $C_{i}=\left(L_{i}, k b_{i}, b r_{i}\right)$, where $L_{i}=\left(\mathbf{K B}_{i}, \mathbf{B S}_{i}, \mathbf{A C C}_{i}\right)$ is an abstract logic, $k b_{i} \in \mathbf{K B}_{i}$ is a knowledge base of $L_{i}$, and br $r_{i}$ is a set of $L_{i}$-bridge rules over $\mathcal{L}=$ $\left(L_{1}, \ldots, L_{n}\right)$ and $1 \leq i \leq n$. 
We usually abbreviate a context $(L, k b, b r)$ as $(k b, b r)$ if the underlying logic $L$ is clear from the context, unless explicitly stated otherwise. A belief state (of $\mathcal{L}$ ) is a sequence $\mathcal{S}=\left(S_{1}, \ldots, S_{n}\right)$ such that $S_{i}$ is an element of $\mathbf{B S}_{i}$ for every $i(1 \leq i \leq n)$. By $\mathbb{B S}_{\mathcal{L}}$ we denote the belief state space of $\mathcal{L}$, i.e., $\mathbb{B S}_{\mathcal{L}}=\{\mathcal{S} \mid \mathcal{S}$ is a belief state of $\mathcal{L}\}$. For a collection $\mathbb{M} \subseteq \mathbb{B S}_{\mathcal{L}}$ of belief states, we denote $\overline{\mathbb{M}}$ the complement of $\mathbb{M}$, i.e., $\overline{\mathbb{M}}=\mathbb{B S}_{\mathcal{L}} \backslash \mathbb{M}$. The belief state $\mathcal{S}$ satisfies an indexed atom $(c: p)$, written $\mathcal{S}=(c: p)$, if $p \in S_{c}$. A bridge rule $r$ of the form (1) is applicable w.r.t. $\mathcal{S}$ if $\mathcal{S}$ satisfies $\left(c_{i}: p_{i}\right)$ for every $i(1 \leq i \leq m)$ and it does not satisfy $\left(c_{j}: p_{j}\right)$ for every $j(m+1 \leq j \leq k)$. By $a p p(b r, \mathcal{S})$ we denote the set of bridge rules in $b r$ that are applicable w.r.t. $\mathcal{S}$.

Definition 2. A belief state $\mathcal{S}=\left(S_{1}, \ldots, S_{n}\right)$ of $\mathcal{L}$ is an equilibrium of $\alpha$ iff $S_{i} \in$ $\mathbf{A C C}_{i}\left(k b_{i} \cup\left\{\operatorname{Head}(r) \mid r \in \operatorname{app}\left(b r_{i}, \mathcal{S}\right)\right\}\right)$ for every $i(1 \leq i \leq n)$.

In what follows we denote $E Q(\alpha)$ the set of equilibria of the multi-context system $\alpha$. If $E Q(\alpha) \neq \emptyset$ then it is consistent, otherwise it is inconsistent. Since we assume that every logic $L_{i}$ in a multi-context system has the ability to express an arbitrary given belief sets by a knowledge base, for any collection $\mathbb{S}$ of belief states of $\mathcal{L}$, there exists a multi-context system $\alpha$ such that $E Q(\alpha)=\mathbb{S}$. For continence, we denote the multicontext system by form $(\mathbb{S})$.

Example 2. [Continued from Example 1] We can use two multi-context systems $\alpha$ and $\beta$ to model the first and second rounds of diagnosis respectively as follows. Here, the underlying logic of each context is propositional logic. The multi-context system $\alpha=$ $\left(C_{1}, C_{2}\right)$ where $C_{1}$ is for Smith, while $C_{2}$ for Alice, $C_{i}=\left(L_{i}, k b_{i}, b r_{i}\right)$ for $i=1,2$,

$-k b_{1}=\left\{\neg b i r d_{-} f l u\right\}$,

- br $r_{1}=\left\{\right.$ bird $f l u \leftarrow(2:$ bird_flue $) ;_{\text {swine }} f l u \leftarrow(2:$ swine_flu $\left.)\right\}$,

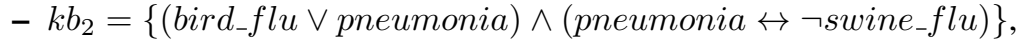

- $b r_{2}=\left\{\right.$ bird_flu $\leftarrow\left(1:\right.$ bird $\left._{-} f l u\right) ; \quad$ swine $f l u \leftarrow\left(1:\right.$ swine $\left.\left._{-} f l u\right)\right\}$.

Please note here that the signature of $L_{1}$ does not contain pneumonia. It is not difficult to check that $\alpha$ has a unique equilibrium $(\emptyset,\{$ pneumonia $\})$.

The multi-context system $\beta=\left(C_{1}^{\prime}, C_{2}^{\prime}\right)$, where $C_{1}^{\prime}$ is for Smith, while $C_{2}^{\prime}$ for Alice, $C_{i}^{\prime}=\left(L_{i}, k b_{i}^{\prime}, b r_{i}^{\prime}\right)$ for $i=1,2$, and

- $k b_{1}^{\prime}=\left\{b i r d_{-} f l u \vee\right.$ swine_flu $\}, b r_{1}^{\prime}=b r_{1}$,

- $k b_{2}^{\prime}=k b_{2}, b r_{2}^{\prime}=b r_{2}$.

One can verify that $\beta$ has two equilibria $\left(S_{1}, S_{2}\right)$ and $\left(S_{1}^{\prime}, S_{2}^{\prime}\right)$ where

- $S_{1}=\{$ bird_flu $\}, S_{2}=\{$ bird_flu,pneumonia $\}$,

- $S_{1}^{\prime}=\{$ bird_flu, swine $f l u\}, S_{2}^{\prime}=\{$ bird_flu, swine $f l u\}$.

Now the problem in Example 1 becomes that how should we change the multi-context system $\alpha$ using $\beta$ ? Though $k b_{1} \wedge k b_{1}^{\prime}$ is obviously consistent, it is a wishful thinking to change only $k b_{1}$ with $k b_{1}+k b_{1}^{\prime}$ in the multi-context system $\alpha$, because both $\alpha$ and $\beta$ have some acceptable belief states (equilibria) but such a revised result has no acceptable belief state. This violates the traditional intuition of belief revision. 


\section{Belief Change for Nonmonotonic Multi-Context Systems}

In the following we consider the three major belief changes, expansion, revision and contraction in multi-context systems, starting from the expansion. We assume that the underlying logical language of multi-context systems is $\mathcal{L}=\left(L_{1}, \ldots, L_{n}\right)$ and belief change operators are mappings of the form $\mathcal{L} \times \mathcal{L} \rightarrow \mathcal{L}$.

\subsection{Expansion}

Recall that the intuition of expansion is to add belief without checking inconsistency. Semantically speaking, for the expansion of $\alpha$ by $\beta$, written $\alpha+\beta$, the acceptable belief states of the expansion result are exactly those that are acceptable belief states of both $\alpha$ and $\beta$. This motivates the postulates for exponion in multi-context systems:

(mcs-E1) $E Q(\alpha+\beta) \subseteq E Q(\alpha) \cap E Q(\beta)$;

(mcs-E2) If $E Q(\alpha) \subseteq E Q(\beta)$ then $E Q(\alpha+\beta)=E Q(\alpha)$;

(mcs-E3) If $E Q\left(\alpha^{\prime}\right) \subseteq E Q(\alpha)$ then $E Q\left(\alpha^{\prime}+\beta\right) \subseteq E Q(\alpha+\beta)$.

Recall that $E Q(\alpha)$ is the set of the equilibria of $\alpha$.

Theorem 1. The expansion operator + satisfies $(m c s-E 1)-(m c s-E 3)$ iff $E Q(\alpha+\beta)=$ $E Q(\alpha) \cap E Q(\beta)$ for any multi-context systems $\alpha$ and $\beta$.

Proof sketch: We prove "only if". By (mcs-E1) we obtain $E Q(\alpha+\beta) \subseteq E Q(\alpha) \cap$ $E Q(\beta)$. Let $\gamma$ be an MCS satisfying $E Q(\gamma)=E Q(\alpha) \cap E Q(\beta)$. By (mcs-E2) we have $E Q(\gamma+\beta)=E Q(\gamma)$. Note that $E Q(\gamma+\beta) \subseteq E Q(\alpha+\beta)$ holds by (mcs-E3). It follows that $E Q(\alpha) \cap E Q(\beta)=E Q(\gamma+\beta) \subseteq E Q(\alpha+\beta)$.

It shows that the expansion of two multi-context systems are semantically unique. For the multi-context systems $\alpha$ and $\beta$ in Example 2, they have no common equilibria. It is not appropriate to model the belief change by the expansion, in the sense that the expansion result is inconsistent.

Recall that, in propositional logic, the expansion of a formula $\alpha$ by a formula $\beta$ can be obtained by $\alpha \wedge \beta$. As the underlying logics in multi-context systems may be nonmonotonic, such a simple approach does not work for multi-context systems. For instance in ASP logic, let $\alpha=\{p \leftarrow \operatorname{not} p\}$ and $\beta=\{p\}$, here $\alpha$ and $\beta$ can be taken as multi-context systems with only one context and having no bridge rules. Then $\alpha \cup \beta=\{p \leftarrow$ not $p ; \quad p\}$ which has an answer set $\{p\}$ but it is not an answer set of $\alpha$, which has no answer set. It illustrates that an expansion for nonmonotonic logics, and thus multi-context systems, is fundamentally different from that of propositional logic.

However, if the expansion can be relaxed as $E Q(\alpha) \cap E Q(\beta) \subseteq E Q(\alpha+\beta)$, i.e., the postulate (mcs-E1) is discarded, then we can have a simple expansion like the one for propositional logic. We will make this point clear in the following. A logic $L$ is quasi-cumulative if for any two knowledge bases $k b$ and $k b^{\prime}$ of $L$, and a belief set $S$ of $L$ such that $S \in \mathbf{A} \mathbf{C C}_{L}(k b) \cap \mathbf{A} \mathbf{C C} C_{L}\left(k b^{\prime}\right)$ then $S \in \mathbf{A} \mathbf{C C}_{L}\left(k b \cup k b^{\prime}\right)$. It is clear that propositional logic, first-order logic and description logic are all semi-cumulative. We can show that ASP logic define in [14] is quasi-cumulative. 
Proposition 1. Let $\Pi_{1}$ and $\Pi_{2}$ be two propositional theories and $S$ is an answer set of $\Pi_{1}$ and $\Pi_{2}$. Then $S$ is an answer set of $\Pi_{1} \cup \Pi_{2}$.

Let $\alpha=\left(C_{1}, \ldots, C_{n}\right)$ and $\beta=\left(C_{1}^{\prime}, \ldots, C_{n}^{\prime}\right)$ be two multi-context systems over the logic languages $\mathcal{L}=\left(L_{1}, \ldots, L_{n}\right)$, where the underlying logics $L_{i} s$ of the contexts are quasi-cumulative. The semi-expansion of $\alpha$ by $\beta$, written $\alpha \mp \beta$, is the multi-context system $\left(C_{1}+C_{1}^{\prime}, \ldots, C_{n}+C_{n}^{\prime}\right)$ where $C_{i}+C_{i}^{\prime}$ is the context whose knowledge base (resp. bridge rule) is the union of the knowledge bases (resp. bridge rules) from the contexts $C_{i}$ and $C_{i}^{\prime}$ for each $i(1 \leq i \leq n)$.

Theorem 2. Let $\alpha$ and $\beta$ be two multi-context systems where the underlying logics of the contexts are quasi-cumulative. If $\mathcal{S}$ is an equilibrium of $\alpha$ and $\beta$ then it is an equilibrium of $\alpha \mp \beta$.

Proof sketch: $\quad$ Let $\mathcal{S}=\left(S_{1}, \ldots, S_{n}\right)$. It suffices to prove $S_{i} \in \mathbf{A C C}_{L_{i}}\left(k b_{i} \cup k b_{i}^{\prime} \cup\right.$ $\left.\left\{\operatorname{Head}(r) \mid r \in \operatorname{app}\left(b r_{i}, \mathcal{S}\right) \cup \operatorname{app}\left(b r_{i}^{\prime}, \mathcal{S}\right)\right\}\right)$ for every $i(1 \leq i \leq n)$. Recall that $S_{i} \in$ $\mathbf{A C C}_{L_{i}}\left(k b_{i} \cup\left\{\operatorname{Head}(r) \mid r \in \operatorname{app}\left(b r_{i}, \mathcal{S}\right)\right\}\right)$ and $S_{i} \in \mathbf{A} \mathbf{C C}_{L_{i}}\left(k b_{i}^{\prime} \cup\{\operatorname{Head}(r) \mid r \in\right.$ $\left.\left.\operatorname{app}\left(b r_{i}^{\prime}, \mathcal{S}\right)\right\}\right)$. By the quasi-cumulative property of the underlying logic $L_{i}$, we have $S_{i} \in \mathbf{A C C}_{L_{i}}\left(k b_{i} \cup\left\{\operatorname{Head}(r) \mid r \in \operatorname{app}\left(b r_{i}, \mathcal{S}\right)\right\} \cup k b_{i}^{\prime} \cup\left\{\operatorname{Head}(r) \mid r \in \operatorname{app}\left(b r_{i}^{\prime}, \mathcal{S}\right)\right\}\right.$, i.e. $S_{i} \in \mathbf{A C C}_{L_{i}}\left(k b_{i} \cup k b_{i}^{\prime} \cup\left\{\operatorname{Head}(r) \mid r \in a p p\left(b r_{i} \cup b r_{i}^{\prime}, \mathcal{S}\right)\right\}\right)$.

\subsection{Revision}

Note that, in the revision of a knowledge base $\alpha$ (a set of formulas) by a formula $\beta$, the intent is that the resulted knowledge base contains $\beta$ and is consistent (unless $\beta$ is not), while keeping whatever information of $\alpha$ can be "reasonably" retained [1]. Semantically speaking, the revision of $\alpha$ by $\beta$ is a knowledge base $\alpha^{\prime}$ whose intended models are just those of $\beta$ that are "closest" to those of $\alpha$ [17]. This motivates the following revision postulates for multi-context systems:

(mcs-R1) $E Q(\alpha+\beta) \subseteq E Q(\beta)$;

(mcs-R2) If $E Q(\alpha) \cap E Q(\beta) \neq \emptyset$ then $E Q(\alpha+\beta)=E Q(\alpha) \cap E Q(\beta)$;

(mcs-R3) If $E Q(\beta) \neq \emptyset$ then $E Q(\alpha+\beta) \neq \emptyset$;

(mcs-R4) If $E Q\left(\alpha_{1}\right)=E Q\left(\alpha_{2}\right)$ and $E Q\left(\beta_{1}\right)=E Q\left(\beta_{2}\right)$

then $E Q\left(\alpha_{1}+\beta_{1}\right)=E Q\left(\alpha_{2}+\beta_{2}\right)$;

(mcs-R5) $E Q\left(\alpha+\beta_{1}\right) \cap E Q\left(\beta_{2}\right) \subseteq E Q\left(\alpha+\beta^{\prime}\right)$;

(mcs-R6) If $E Q\left(\alpha \dot{+} \beta_{1}\right) \cap E Q\left(\beta_{2}\right) \neq \emptyset$ then $E Q\left(\alpha \dot{+} \beta^{\prime}\right) \subseteq E Q\left(\alpha \dot{+} \beta_{1}\right) \cap E Q\left(\beta_{2}\right)$

where $\beta^{\prime}$ is a multi-context system such that $E Q\left(\beta^{\prime}\right)=E Q\left(\beta_{1}\right) \cap E Q\left(\beta_{2}\right)$. The intended meaning of these revision postulates for multi-context systems is similar to that of propositional logic in Section 2.1.

Orders between belief states. Let $L$ be an abstract logic. A pre-order $\preceq_{L}$ over the possible belief sets ${ }^{3}$ of $L$ is a reflexive and transitive binary relation on $\mathbf{B S}_{L}$. And we define $\prec_{L}$ as $S \prec_{L} S^{\prime}$ iff $S \preceq_{L} S^{\prime}$ and $S^{\prime} \swarrow_{L} S$. A pre-order $\preceq_{L}$ is total if for every two belief sets $S$ and $S^{\prime}$ of $L$, either $S \preceq_{L} S^{\prime}$ or $S^{\prime} \preceq_{L} S$. A function that assigns a knowledge base $k b$ of $L$ a pre-order $\preceq_{k b}$ is faithful if the following conditions hold:

\footnotetext{
${ }^{3}$ Recall that the notion of belief sets corresponds to "interpretations" in propositional logic.
} 
(1) If $S, S^{\prime} \in \mathbf{A C C}_{L}(k b)$ then $S \preceq_{k b} S^{\prime}$ does not hold.

(2) If $S \in \mathbf{A C C}_{L}(k b)$ and $S^{\prime} \notin \mathbf{A C C} \mathbf{C C}_{L}(k b)$ then $S \preceq_{k b} S^{\prime}$.

(3) If $\mathbf{A C C}_{L}(k b)=\mathbf{A} \mathbf{C C}_{L}\left(k b^{\prime}\right)$ then $\preceq_{k b}$ is same to $\preceq_{k b^{\prime}}$.

Let $\mathbb{M}_{L}$ be a collection of belief sets of $L$. A belief set $S \in \mathbb{M}_{L}$ is minimal w.r.t. $\preceq_{k b}$ if there is no $S^{\prime} \in \mathbb{M}_{L}$ such that $S^{\prime} \prec_{k b} S$. We denote

$$
\operatorname{Min}\left(\mathbb{M}_{L}, \preceq_{k b}\right)=\left\{S \mid S \text { is minimal in } \mathbb{M}_{L} \text { w.r.t. } \preceq_{k b}\right\} \text {. }
$$

Let $\alpha=\left(C_{1}, \ldots, C_{n}\right)$ be an MCS over langues $\mathcal{L}=\left(L_{1}, \ldots, L_{n}\right)$ with $C_{i}=$ $\left(L_{i}, k b_{i}, b r_{i}\right)(1 \leq i \leq n), \mathcal{S}=\left(S_{1}, \ldots, S_{n}\right)$ and $\mathcal{S}^{\prime}=\left(S_{1}^{\prime}, \ldots, S_{n}^{\prime}\right)$ two belief states of $\alpha$. A pre-order $\preceq_{\mathcal{L}}$ over the space of belief states of $\mathcal{L}$ is a reflexive and transitive binary relation such that $\mathcal{S} \preceq_{\mathcal{L}} \mathcal{S}^{\prime}$ if $S_{i} \preceq_{L_{i}} S_{i}^{\prime}$ for every $i(1 \leq i \leq n)$. Similarly, $\prec_{\mathcal{L}}$ is defined as $\mathcal{S} \prec_{\mathcal{L}} \mathcal{S}^{\prime}$ if $\mathcal{S} \preceq_{\mathcal{L}} \mathcal{S}^{\prime}$ and $\mathcal{S}^{\prime} \swarrow_{\mathcal{L}} \mathcal{S}$. A pre-order $\preceq_{\mathcal{L}}$ is total if either $\mathcal{S} \preceq_{\mathcal{L}} \mathcal{S}^{\prime}$ or $\mathcal{S}^{\prime} \preceq_{\mathcal{L}} \mathcal{S}$ for every two of belief states $\mathcal{S}$ and $\mathcal{S}^{\prime}$ of $\mathcal{L}$. A function that assigns $\alpha$ a pre-order $\preceq_{\alpha}$ is faithful whenever the assigned pre-order $\preceq_{k b_{i}}$ is faithful for every $i(1 \leq i \leq n)$.

Let $\mathbb{S}$ be a collection of belief states of $\mathcal{L}$. A belief state $\mathcal{S} \in \mathbb{S}$ is minimal w.r.t. $\preceq_{\alpha}$ if there is no $\mathcal{S}^{\prime} \in \mathbb{S}$ such that $\mathcal{S}^{\prime} \prec_{\alpha} \mathcal{S}$. We define

$$
\operatorname{Min}\left(\mathbb{S}, \preceq_{\alpha}\right)=\left\{\mathcal{S} \mid \mathcal{S} \text { is minimal in } \mathbb{S} \text { w.r.t. } \preceq_{\alpha}\right\} .
$$

Theorem 3. A revision operator $\dot{+}$ satisfies the conditions (mcs-RI)-(mcs-R6) iff there exists a faithful assignment that maps each MCS $\alpha$ of $\mathcal{L}$ to a total pre-order $\preceq_{\alpha}$ such that $E Q(\alpha+\beta)=\operatorname{Min}\left(E Q(\beta), \preceq_{\alpha}\right)$.

Proof sketch: The overall proof is similar to that of Theorem 3.3 of [17]. We outline the proof of "only if". Firstly we define $\preceq_{\alpha}$ over the space of belief states as $\mathcal{S} \preceq{ }_{\alpha} \mathcal{S}^{\prime}$ if either $\mathcal{S} \in E Q(\alpha)$ or $\mathcal{S} \in E Q\left(\alpha+\beta^{\prime}\right)$ where $\beta^{\prime}$ is an MCS such that $E Q\left(\beta^{\prime}\right)=\left\{\mathcal{S}, \mathcal{S}^{\prime}\right\}$. We can show that $\preceq_{\alpha}$ is a total pre-order and the assignment that maps $\alpha$ to $\preceq_{\alpha}$ is faithful. Finally we can prove $E Q(\alpha \dot{+} \beta) \subseteq \operatorname{Min}\left(E Q(\beta), \preceq_{\alpha}\right)$ and $\operatorname{Min}\left(E Q(\beta), \preceq_{\alpha}\right) \subseteq E Q(\alpha+\beta)$.

This theorem shows that our belief revision operator for multi-context systems obeys the principle of minimal change in the sense that the acceptable belief states (equilibria) of the revision result $\alpha \dot{+} \beta$ are exactly the ones of $\beta$ that are "closet" to those of $\alpha$. There are many approaches of "closeness" in propositional logic, we consider two distance-based operators for multi-context systems in the following.

Two distance based revision operators. Given two belief states $\mathcal{S}=\left(S_{1}, \ldots, S_{n}\right)$ and $\mathcal{S}^{\prime}=\left(S_{1}^{\prime}, \ldots, S_{n}^{\prime}\right)$ of $\mathcal{L}$, we define the following notations,

- $|\mathcal{S}|=\sum_{i=1}^{n}\left|S_{i}\right|$, where $|S|$ denotes the cardinality of the set $S$,

- $\mathcal{S} \subseteq \mathcal{S}^{\prime}$ if $S_{i} \subseteq S_{i}^{\prime}$ for every $i(1 \leq i \leq n)$;

- $\mathcal{S} \subset \mathcal{S}^{\prime}$ if $\mathcal{S} \subseteq \mathcal{S}^{\prime}$ and $S_{i} \subset S_{i}^{\prime}$ for some $i(1 \leq i \leq n)$;

- $\mathcal{S} \ominus \mathcal{S}^{\prime}=\left(S_{1} \ominus S_{1}^{\prime}, \ldots, S_{n} \ominus S_{n}^{\prime}\right)$, where $\ominus$ is the symmetric difference operator between sets, i.e. $X \ominus Y=(X \backslash Y) \cup(Y \backslash X)$. 
Let $\alpha$ and $\beta$ be two multi-context systems. We define

$$
\begin{array}{r}
\ominus^{\min }(\alpha, \beta)=\min _{\subseteq}\left(\left\{\mathcal{S} \ominus \mathcal{S}^{\prime}: \mathcal{S} \in E Q(\alpha) \& \mathcal{S}^{\prime} \in E Q(\beta)\right\}\right), \\
|\ominus|^{m i n}(\alpha, \beta)=\min _{\leq}\left(\left\{\left|\mathcal{S} \ominus \mathcal{S}^{\prime}\right|: \mathcal{S} \in E Q(\alpha) \& \mathcal{S}^{\prime} \in E Q(\beta)\right\}\right)
\end{array}
$$

where $\min _{\subseteq}(\mathbb{X})$ denotes the set of minimal (under set inclusion) elements in collection $\mathbb{X}$ of sets, and $\min _{\leq}(X)$ denotes the least number in the set $X$ of numbers.

Definition 3. The revision operators $\dot{+}_{s}$ and $\dot{+}_{d}$ for multi-context systems are defined respectively as follows:

$$
\begin{array}{r}
E Q\left(\alpha \dot{+}_{s} \beta\right)=\left\{\mathcal{S} \in E Q(\beta) \mid \exists \mathcal{S}^{\prime} \in E Q(\alpha) \text { s.t. } \mathcal{S} \ominus \mathcal{S}^{\prime} \in \ominus^{\min }(\alpha, \beta)\right\}, \\
E Q\left(\alpha \dot{+}_{d} \beta\right)=\left\{\mathcal{S} \in E Q(\beta) \mid \exists \mathcal{S}^{\prime} \in E Q(\alpha) \text { s.t. }\left|\mathcal{S} \ominus \mathcal{S}^{\prime}\right|=|\ominus|^{m i n}(\alpha, \beta)\right\} .
\end{array}
$$

Intuitively, the revision result $\alpha \dot{+}_{\star} \beta$ has the equilibria of $\beta$ that are "closest" to some equilibrium of $\alpha$, where $\star \in\{s, d\}$. One can see that the operator $\dot{+}_{s}$ (resp. $\dot{+}_{d}$ ) is identical to Sato revision operator $\circ_{S}$ (resp. Dalal revision operator $\circ_{D}$ ) in [17] when there is only one context with underlying proportional logic and without bridge rules. Therefore, the revision operators $\dot{+}_{s}$ and $\dot{+}_{d}$ are generalizations of propositional knowledge base revision operator $\circ_{S}$ and $\circ_{D}$ respectively.

Example 3 (Continued from Example 2). According to the operators $\dot{+}_{s}$ and $\dot{+}_{d}$, one can check that the unique equilibrium of $\alpha \dot{+}_{\star} \beta$ is $\left(S_{1}, S_{2}\right)$, where $S_{1}=\{$ bird_flu $\}$, $S_{2}=\{$ bird_flu,pneumonia $\}$ and $\star \in\{s, d\}$. A multi-context system corresponding to $\alpha \dot{+}_{\star} \beta$ can be $\left(C_{1}^{\prime \prime}, C_{2}^{\prime \prime}\right)$ with $C_{i}^{\prime \prime}=\left(k b_{i}^{\prime \prime}, b r_{i}^{\prime \prime}\right)$ for $i=1,2$ where

- $k b_{1}^{\prime \prime}=\left\{\right.$ bird_flu $\wedge \neg$ swine_flu\}, note that pneumonia is not a symbol of $L_{1}$,

- $k b_{2}^{\prime \prime}=\{$ bird_flu $\wedge$ pneumonia $\wedge \neg$ swine_flu $\}$,

- $b r_{1}^{\prime \prime}=b r_{2}^{\prime \prime}=\emptyset$, or alternatively $b r_{1}^{\prime \prime}=b r_{1}$ and $b r_{2}^{\prime \prime}=b r_{2}$.

Theorem 4. Assume $\alpha$ is consistent. The revision operators $\dot{+}_{s}$ satisfies (mcs-R1) (mcs-R5); and the revision operator $\dot{+}_{d}$ satisfies (mcs-R1)-(mcs-R6).

Proof sketch: We prove that $\dot{+}_{d}$ satisfies (mcs-R6). By $E Q\left(\beta^{\prime}\right)=E Q\left(\beta_{1}\right) \cap E Q\left(\beta_{2}\right)$, we have $|\ominus|^{\min }\left(\alpha, \beta_{1}\right) \leq|\ominus|^{\min }\left(\alpha, \beta^{\prime}\right)$. Recall that $E Q\left(\alpha \dot{+}_{d} \beta_{1}\right) \cap E Q\left(\beta_{2}\right) \neq \emptyset$. It implies that there exists $\mathcal{S} \in E Q\left(\beta_{2}\right) \cap E Q\left(\beta_{1}\right)$ and $\left|\mathcal{S} \ominus \mathcal{S}^{\prime}\right|=|\ominus|^{\min }\left(\alpha, \beta_{1}\right)$ for some $\mathcal{S}^{\prime} \in E Q(\alpha)$, from which it follows that $|\ominus|^{\min }\left(\alpha, \beta^{\prime}\right) \leq|\ominus|^{\min }\left(\alpha, \beta_{1}\right)$. Thus $|\ominus|^{\text {min }}\left(\alpha, \beta^{\prime}\right)=|\ominus|^{\text {min }}\left(\alpha, \beta_{1}\right)$. Let $\mathcal{S} \in E Q\left(\alpha \dot{+}_{d} \beta^{\prime}\right)$. We have $\mathcal{S} \in E Q\left(\beta_{1}\right) \cap$ $E Q\left(\beta_{2}\right)$ and $\exists \mathcal{S}^{\prime} \in E Q(\alpha)$ s.t. $\left|\mathcal{S} \ominus \mathcal{S}^{\prime}\right|=|\ominus|^{\min }\left(\alpha, \beta^{\prime}\right)$. It implies $\mathcal{S} \in E Q\left(\beta_{1}\right)$ and $\mathcal{S}^{\prime} \in E Q(\alpha)$ s.t. $\left|\mathcal{S} \ominus \mathcal{S}^{\prime}\right|=|\ominus|^{\min }\left(\alpha, \beta_{1}\right)$. Thus $\mathcal{S} \in E Q\left(\alpha \dot{+}_{d} \beta_{1}\right)$.

The operator $\dot{+}_{s}$ may falsify (mcs-R6) due to the fact that the corresponding revision operator $\circ_{S}$ in propositional logic may falsify the corresponding postulate (R6) (cf. Example 4.1 of [17]).

Relating to classical revision via loop formulas. Recall that every context in a multicontext system has its own language, which has its own signature too. In this sense, the signatures for contexts are pairwise disjoint. In the following we assume that the signatures for the langues in $\mathcal{L}$ share no common symbols, unless explicitly stated otherwise. 
In the following readers are assumed being familiar with answer set programming [15] and the basic notions of loops and loop formulas of logic programs [20].

We note that if $\mathcal{L}$ consists of ASP logics, then every MCS can be translated into a propositional theory via loop formulas [7]. Briefly, given a multi-context system $\alpha=$ $\left(C_{1}, \ldots, C_{n}\right)$ with $C_{i}=\left(L_{i}, k b_{i}, b r_{i}\right)$ where $L_{i} s$ are ASP logics, by identifying $C_{i}$ with the logic program $\Pi_{i}=k b_{i} \cup \ell\left(b r_{i}\right)$ where $\ell\left(b r_{i}\right)$ is obtained from $b r_{i}$ by replacing every indexed atom of the form $(n: p)$ with the atom $p$,

- the loops of $C_{i}$ are the loops of $\Pi_{i},{ }^{4}$

- the loop formula $\lambda\left(X, C_{i}\right)$ for loop $X$ of $C_{i}$ is the same as the loop formula $L F\left(X, \Pi_{i}\right)$ for the loop $X$ of $\Pi_{i}$ with the exception that it allows for circular supports ${ }^{5}$ from $\ell\left(b r_{i}\right)$,

- the loop completion $\pi\left(C_{i}\right)$ of $C_{i}$ is the following theory

$$
\left\{\lambda\left(X, C_{i}\right) \mid X \text { is a loop of } C_{i}\right\} \cup \kappa\left(\Pi_{i}\right) .
$$

where $\kappa\left(\Pi_{i}\right)$ is obtained from $\Pi_{i}$ by replacing every rule as a formula ${ }^{6}$,

- the loop completion $\pi(\alpha)$ of $\alpha$ is $\pi\left(C_{1}\right) \cup \cdots \cup \pi\left(C_{n}\right)$.

It is proved that the equilibria of $\alpha$ correspond one-to-one to the models of $\pi(\alpha)$ (cf. Theorem 5 of [7]). To illustrate the notion of loop formulas for multi-context systems, let us consider the following example.

Example 4. Let $\alpha=\left(C_{1}, C_{2}\right)$ and $\beta=\left(C_{1}^{\prime}, C_{2}^{\prime}\right)$ be two multi-context systems with $C_{i}=\left(L_{i}, k b_{i}, b r_{i}\right)$ and $C_{i}^{\prime}=\left(L_{i}, k b_{i}^{\prime}, b r_{i}^{\prime}\right)$ for $i=1,2$ where

- both $L_{1}$ and $L_{2}$ are ASP logics,

- $k b_{1}=\{p\}, k b_{2}=\left\{p^{\prime}\right\}, k b_{1}^{\prime}=\{\leftarrow p\}$ and $k b_{2}^{\prime}=\left\{\leftarrow p^{\prime}\right\}$,

- br $r_{1}=\left\{p \leftarrow \operatorname{not}\left(2: p^{\prime}\right)\right\}, b r_{2}=\left\{p^{\prime} \leftarrow(1: p)\right\}, b r_{1}^{\prime}=b r_{2}^{\prime}=\emptyset$.

One can check that the unique equilibrium of $\alpha$ is $\mathcal{S}=\left(\{p\},\left\{p^{\prime}\right\}\right)$ and the unique equilibrium of $\beta$ is $\mathcal{S}^{\prime}=(\emptyset, \emptyset)$. Note that the loop of $C_{1}$ is $X=\{p\}$, which is the unique loop of $C_{1}^{\prime}$, and $X^{\prime}=\left\{p^{\prime}\right\}$ is the unique loop of $C_{2}$ and $C_{2}^{\prime}$. Now we have:

- $\pi\left(C_{1}\right) \equiv p \wedge\left(\neg p^{\prime} \supset p\right) \wedge\left(p \supset \top \vee \neg p^{\prime}\right)$, the last conjunct is $\lambda(X, \alpha)$,

- $\pi\left(C_{2}\right) \equiv p^{\prime} \wedge\left(p \supset p^{\prime}\right) \wedge\left(p^{\prime} \supset \top \vee p\right)$, the last conjunct is $\lambda\left(X^{\prime}, \beta\right)$,

- $\pi(\alpha) \equiv \pi\left(C_{1}\right) \wedge \pi\left(C_{2}\right) \equiv p \wedge p^{\prime}$,

- $\pi\left(C_{1}^{\prime}\right) \equiv \neg p \wedge(p \supset \perp)$, the last conjunct is $\lambda\left(X, C_{1}^{\prime}\right)$,

- $\pi\left(C_{2}^{\prime}\right) \equiv \neg p^{\prime} \wedge\left(p^{\prime} \supset \perp\right)$, the last conjunct is $\lambda\left(X^{\prime}, C_{2}^{\prime}\right)$,

$-\pi(\beta) \equiv \neg p \wedge \neg p^{\prime}$.

One can verify that, over the signature $\left\{p, p^{\prime}\right\}$, the unique model of $\pi(\alpha)$ is $\left\{p, p^{\prime}\right\}$, and the unique model of $\pi(\beta)$ is $\emptyset$.

We will show that belief revision in multi-context systems with ASP logics can be achieved by revision in propositional logic via loop formulas.

\footnotetext{
${ }^{4}$ Here, every single element set is a loop as defined in [18].

${ }^{5}$ E.g., the rule " $a \leftarrow a, b$, not $c$ " is a circular supporting rule for $a$.

${ }^{6}$ E.g., the rule " $a \leftarrow b, c$, not $d$ " is replaced by $\neg d \wedge b \wedge c \supset a$.
} 
Lemma 1. Let $\mathcal{S}=\left(S_{1}, \ldots, S_{n}\right)$ and $\mathcal{S}^{\prime}=\left(S_{1}^{\prime}, \ldots, S_{n}^{\prime}\right)$ be two belief states of $\mathcal{L}$.

(i) $\mathcal{S} \subseteq \mathcal{S}^{\prime}$ iff $\left(\bigcup_{1<i<n} S_{i}\right) \subseteq\left(\bigcup_{1<i<n} S_{i}^{\prime}\right)$.

(ii) $|\mathcal{S}| \leq\left|\mathcal{S}^{\prime}\right|$ iff $\left|\left(\bigcup_{1 \leq i \leq n} S_{i}\right)\right| \leq\left\lceil\left(\bigcup_{1 \leq i \leq n} S_{i}^{\prime}\right) \mid\right.$.

The following theorem shows that, a belief revision for multi-context systems with loop definable logics can be achieved via a belief revision in propositional logic.

Theorem 5. Let $\alpha$ and $\beta$ be two multi-context systems where the underlying logics are ASP. Then the equilibria of $\alpha \dot{+}_{s} \beta$ (resp. $\alpha \dot{+}_{d} \beta$ ) correspond one-to-one to the models of $\pi(\alpha) \circ_{S} \pi(\beta)$ (resp. $\pi(\alpha) \circ_{D} \pi(\beta)$ ).

Proof sketch: We prove the case $\dot{+}_{s}$. Let $S=\left(S_{1}, \ldots, S_{n}\right)$ be a belief state of $\mathcal{L}$. We define $\pi(\mathcal{S})=\bigcup_{1<i<n} S_{i}$. Clearly, $\pi$ is a one-to-one mapping from $\mathcal{S}$ to $\pi(\mathcal{S})$. We have that $\mathcal{S} \in E Q\left(\alpha+{ }_{s} \beta\right)$

iff $\mathcal{S} \in E Q(\beta)$ and $\exists \mathcal{S}^{\prime} \in E Q(\alpha)$ s.t $\mathcal{S} \ominus \mathcal{S}^{\prime} \in \ominus^{\min }(\alpha, \beta)$

iff $\pi(\mathcal{S}) \in \operatorname{Mod}(\pi(\beta))$ and $\exists \pi\left(\mathcal{S}^{\prime}\right) \in \operatorname{Mod}(\pi(\alpha))$ s.t $\pi(\mathcal{S}) \ominus \pi\left(\mathcal{S}^{\prime}\right) \in \ominus^{\min }(\pi(\alpha), \pi(\beta))$ by (i) of Lemma 1, the fact $\pi(\mathcal{S}) \ominus \pi\left(\mathcal{S}^{\prime}\right)=\pi\left(\mathcal{S} \ominus \mathcal{S}^{\prime}\right)$ and Theorem 5 of [7] iff $\pi(\mathcal{S}) \in \operatorname{Mod}\left(\pi(\alpha) \circ_{s} \pi(\beta)\right)$.

Example 5. [Continued from Example 4] In terms of the revision operators $\dot{+}_{s}$ and $\dot{+}_{d}$, we evidently have that the unique equilibrium of $\alpha \dot{+}_{s} \beta$ and $\alpha \dot{+}_{d} \beta$ is $\mathcal{S}^{\prime}$. Thus we can take $\beta$ as one of its revision result. One can check that $\pi(\alpha) \circ_{\star} \pi(\beta)=\left(p \wedge p^{\prime}\right) \circ_{\star}(\neg p \wedge$ $\left.\neg p^{\prime}\right)$, the unique model of $\pi(\alpha) \circ_{\star} \pi(\beta)$ is the model of $\pi(\beta)$ for $\star \in\{S, D\}$.

Partial order revisions. To characterize revision operators for multi-context systems that satisfy the postulates (mcs-R1) - (mcs-R6) in terms of pre-orders among belief states, it requires that every two belief states must be comparable, i.e. the pre-orders are total. Similar to the belief revision for propositional logic, it needs some partial orders among interpretations instead of total ones sometimes. We follow Katsuno and Mendelzon's approach to relax the totality conditions on orders over belief states. This motivates the following two postulates as a replacement of (mcs-R6):

(mcs-R6a) If $E Q\left(\alpha \dot{+} \beta_{1}\right) \subseteq E Q\left(\beta_{2}\right)$ and $E Q\left(\alpha \dot{+} \beta_{2}\right) \subseteq E Q\left(\beta_{1}\right)$ then $E Q\left(\alpha \dot{+} \beta_{1}\right)=$ $E Q\left(\alpha+\beta_{2}\right)$.

(mcs-R6b) $E Q\left(\alpha \dot{+} \beta_{1}\right) \cap E Q\left(\alpha \dot{+} \beta_{2}\right) \subseteq E Q(\alpha \dot{+} \beta)$ where $\beta$ is an MCS such that $E Q(\beta)=E Q\left(\beta_{1}\right) \cup E Q\left(\beta_{2}\right)$.

There are two alternatives to the postulate (mcs-R6a):

(mcs-R6w) If $E Q\left(\alpha \dot{+} \beta_{1}\right) \subseteq E Q\left(\beta_{2}\right)$, then $E Q(\alpha \dot{+} \beta) \subseteq E Q\left(\alpha+\beta_{1}\right) \cap E Q\left(\beta_{2}\right)$ where $\beta$ is an MCS satisfying $E Q(\beta)=E Q\left(\beta_{1}\right) \cap E Q\left(\beta_{2}\right)$.

(mcs-Rt) If $E Q(\alpha \dot{+} \beta)=E Q\left(\beta_{1}\right)$ and $E Q(\alpha \dot{+} \gamma)=E Q\left(\beta_{2}\right)$ then $E Q(\alpha+\zeta)=E Q\left(\beta_{1}\right)$ where $\beta, \gamma, \zeta$ are MCSs satisfying $E Q(\beta)=E Q\left(\beta_{1}\right) \cup E Q\left(\beta_{2}\right), E Q(\gamma)=$ $E Q\left(\beta_{2}\right) \cup E Q\left(\beta_{3}\right)$ and $E Q(\zeta)=E Q\left(\beta_{1}\right) \cup E Q\left(\beta_{3}\right)$.

Lemma 2. Assume that a revision operator + for multi-context systems satisfies (mcsR1) - (mcs-R5). Then the following conditions are equivalent: 
(i) The revision operator $\dot{+}$ satisfies $(m c s-R 6 a)$.

(ii) The revision operator $\dot{+}$ satisfies (mcs-R6w).

(iii) The revision operator $\dot{+}$ satisfies (mcs-Rt).

Theorem 6. A revision operator $\dot{+}$ satisfies conditions $(m c s-R 1)-(m c s-R 5)$ and (mcsR6a)-(mcs-R6b) iff there exists a faithful assignment that maps each multi-context system $\alpha$ to a partial pre-order $\preceq_{\alpha}$ such that $E Q(\alpha+\beta)=\operatorname{Min}\left(E Q(\beta), \preceq_{\alpha}\right)$.

Proof sketch: We prove the "if" part. Suppose that a faithful assignment maps $\alpha$ to a pre-order $\preceq_{\alpha}$. We define a revision operator $\dot{+}$ by $E Q(\alpha \dot{+} \beta)=\operatorname{Min}\left(E Q(\beta), \preceq_{\alpha}\right)$. Similar to Theorem 3, we can prove that $\dot{+}$ satisfies (mcs-R1)-(mcs-R5).

To prove (mcs-R6a), assume that $\mathcal{S} \in E Q\left(\alpha+\beta_{1}\right)$ and $\mathcal{S} \notin E Q\left(\alpha+\beta_{2}\right)$. By $E Q\left(\alpha \dot{+} \beta_{1}\right) \subseteq E Q\left(\beta_{2}\right)$ and (mcs-R1), we obtain $\mathcal{S} \in E Q\left(\beta_{1}\right) \cap E Q\left(\beta_{2}\right)$. Note further that $\mathcal{S}$ is minimal in $E Q\left(\beta_{1}\right)$ w.r.t $\preceq_{\alpha}$ but not minimal in $E Q\left(\beta_{2}\right)$ w.r.t $\preceq_{\alpha}$. The latter implies by (mcs-R3) that $\exists \mathcal{S}^{\prime} \in E Q\left(\beta_{2}\right)$ such that $\mathcal{S}^{\prime}$ is minimal in $E Q\left(\beta_{2}\right)$ w.r.t $\preceq_{\alpha}$ and then $\mathcal{S}^{\prime} \prec_{\alpha} \mathcal{S}$. It follows that $\mathcal{S}^{\prime} \in E Q\left(\beta_{1}\right)$ due to the fact $E Q\left(\alpha \dot{+} \beta_{2}\right) \subseteq E Q\left(\beta_{1}\right)$, a contradiction. To prove (mcs-R6b), let $\mathcal{S} \in E Q\left(\alpha \dot{+} \beta_{1}\right) \cap E Q\left(\alpha+\beta_{2}\right)$. In the case that $\mathcal{S} \notin E Q\left(\alpha+\operatorname{form}\left(E Q\left(\beta_{1}\right) \cup E Q\left(\beta_{2}\right)\right)\right)$, there exists $\mathcal{S}^{\prime} \in E Q\left(\beta_{1}\right) \cup E Q\left(\beta_{2}\right)$ such that $\mathcal{S}^{\prime} \prec_{\alpha} \mathcal{S}$. That is impossible since $\mathcal{S}$ is minimal in $E Q\left(\beta_{1}\right)$ w.r.t $\preceq_{\alpha}$ and it is minimal in $E Q\left(\beta_{2}\right)$ w.r.t $\preceq_{\alpha}$.

\subsection{Contraction}

In propositional logic belief contraction is definable via Harper identity. It has independently desirable properties. In the following we adapt these properties for nonmonotonic multi-context systems and relate it with the revision and expansion for MCSs.

Suppose - is a contraction operator for multi-context systems. The contraction postulates for multi-context systems are formulated below from a model theoretical view:

(mcs-C1) $E Q(\alpha) \subseteq E Q(\alpha \dot{-\beta})$;

(mcs-C2) If $E Q(\alpha) \nsubseteq E Q(\beta)$ then $E Q(\alpha \dot{-} \beta)=E Q(\alpha)$;

(mcs-C3) If $\beta$ is not a tautology then $E Q(\alpha \dot{-} \beta) \nsubseteq E Q(\beta)$;

(mcs-C4) If $E Q(\beta)=E Q(\gamma)$ then $E Q(\alpha \dot{-} \beta)=E Q(\alpha \dot{-} \gamma)$;

(mcs-C5) $E Q(\alpha \dot{-} \beta) \cap E Q(\beta) \subseteq E Q(\alpha)$;

(mcs-C6) $E Q(\alpha \dot{-} \beta) \subseteq E Q\left(\alpha \dot{-} \beta_{1}\right) \cup E Q\left(\alpha \dot{-} \beta_{2}\right)$;

(mcs-C7) $E Q\left(\alpha \dot{-} \beta_{1}\right) \subseteq E Q(\alpha \dot{-} \beta)$ whenever $E Q(\alpha \dot{-} \beta) \nsubseteq E Q\left(\beta_{1}\right)$

where $\beta$ is a multi-context system with $E Q(\beta)=E Q\left(\beta_{1}\right) \cap E Q\left(\beta_{2}\right)$ in (mcs-C6) and (mcs-C7). In terms of Harper identity, a belief contraction - can be defined via a belief revision $\dot{+}$ as follows:

$$
E Q(\alpha \dot{-} \beta)=E Q(\alpha) \cup E Q\left(\alpha+\beta^{\prime}\right)
$$

where $\beta^{\prime}$ is a multi-context system satisfying $E Q\left(\beta^{\prime}\right)=\overline{E Q(\beta)}$.

Proposition 2. The belief contraction defined by equation (2) satisfies the postulates (mcs-C1)-(mcs-C7). 
Based on the contraction and the above expansion, the revision operator can be alternatively defined by Levi identity as follows:

$$
E Q\left(\alpha \dot{+}^{\prime} \beta\right)=E Q\left(\left(\alpha \dot{-} \beta^{\prime}\right)+\beta\right)
$$

where $\beta^{\prime}$ is a multi-context system satisfying $E Q\left(\beta^{\prime}\right)=\overline{E Q(\beta)}$.

Proposition 3. The revision operator $\dot{+}^{\prime}$ is identical to $\dot{+}$ for multi-context systems.

\section{Discussion and Conclusion}

Related work. Belief revision has been investigated in multiple (heterogeneous) information sources, multi-agent systems in particular [22,10,11]. On the one hand, there is no AGM style postulates for those approaches; on the other hand, both knowledge bases and bridge rules of agents are monotonic.

Note that each knowledge base in propositional logic can be seen as an MCS. One can easily see that the belief revision operator $\dot{+}$ for multi-context systems is a generalization to the one for propositional logic. As every answer set program can be taken as an MCS, the proposed belief change theory for MCS is applicable for ASP as well. This approach is substantially different from the existing ones, however. Many of them are under the title update $[27,2,12,24,8]$, in which a update of a sequence of logic programs is focused and founded on the notion of causal rejection - some rules in a logic program will be rejected since they conflict with some others in a higher priority logic program. Thus these approaches fall outside the AGM belief revision paradigm and are syntactic in nature. Delgrande et al. proposed a model-theoretical belief revision model for ASP [9], in which they concentrated on "SE-models" that capture the strong equivalence $^{7}$ of logic programs [19]. When applying our belief change theory of MCS to ASP, it considers answer sets instead of SE-models.

Conclusion and future work. Following AGM model we proposed a model-theoretical belief expansion, revision and contraction theories for nonmonotonic multi-context systems under equilibria semantics. Two distance-based revision operators were presented. There are two other important semantics - grounded equilibria and well-founded model [3]. We believe that it is possible to establish similar results under the two semantics. Some interesting and challenging issues remain. For instance, in the case that every underlying logics of MCS has a belief revision operator, is it possible to obtain a rational revision operator for MCS by combining these individual revision operators?

\section{References}

1. Carlos E. Alchourrön, Peter Gärdenfors, and David Makinson. On the logic of theory change: Partial meet contraction and revision functions. JSL, 50(2):510-530, 1985.

2. J.J. Alferes, J.A. Leite, L.M. Pereira, H. Przymusinska, and T.C. Przymusinski. Dynamic updates of non-monotonic knowledge bases. JLP, 45(1):43 - 70, 2000.

\footnotetext{
${ }^{7}$ Two logic programs $\Pi_{1}$ and $\Pi_{2}$ are strongly equivalent if $\Pi_{1} \cup \Pi$ and $\Pi_{2} \cup \Pi$ have the same answer sets for any logic program $\Pi$.
} 
3. Gerhard Brewka and Thomas Eiter. Equilibria in heterogeneous nonmonotonic multi-context systems. In AAAI-2007, 385-390, Vancouver, Canada, 2007. AAAI Press.

4. Gerhard Brewka, Thomas Eiter, and Michael Fink. Nonmonotonic multi-context systems: A flexible approach for integrating heterogeneous knowledge sources. V. 6565 of LNCS, 233-258. Springer, 2011.

5. Gerhard Brewka, Thomas Eiter, and Miroslaw Truszczynski. Answer set programming at a glance. Communications of the ACM, 54(12):92-103, 2011.

6. Mukesh Dalal. Investigations into a theory of knowledge base revision. In AAAI-1988, 475-479, St. Paul, MN, 1988. AAAI Press / The MIT Press.

7. Minh Dao-Tran, Thomas Eiter, Michael Fink, and Thomas Krennwallner. Distributed nonmonotonic multi-context systems. In $K R-2010,60-70$, Toronto, 2010. AAAI Press.

8. James P. Delgrande, Torsten Schaub, and Hans Tompits. A preference-based framework for updating logic programs. LPNMR-2007, V. 4483 of LNCS, 71-83. Springer, 2007.

9. James P. Delgrande, Torsten Schaub, Hans Tompits, and Stefan Woltran. A model-theoretic approach to belief change in answer set programming. TOCL, 14(2):A:1-A:42, 2012.

10. Aldo Franco Dragoni and Paolo Giorgini. Toward a revision for multi-context systems. LABS-2002 Tolose (France), 2002.

11. Aldo Franco Dragoni and Paolo Giorgini. Distributed belief revision. Autonomous Agents and Multi-Agent Systems, 6(2):115-143, 2003.

12. Thomas Eiter, Michael Fink, Giuliana Sabbatini, and Hans Tompits. On properties of update sequences based on causal rejection. TPLP, 2:711-767, 102002.

13. Eduardo L. Fermé and Sven Ove Hansson. AGM 25 years - twenty-five years of research in belief change. Journal of Philosophical Logic, 40(2):295-331, 2011.

14. Paolo Ferraris. Logic programs with propositional connectives and aggregates. TOCL, 12(4):25:1-25:40, 2011.

15. Michael Gelfond and Vladimir Lifschitz. The stable model semantics for logic programming. In ICLP-1988, 1070-1080, Seattle, Washington, 1988. MIT Press.

16. Frank Van Harmelen, Vladimir Lifschitz, and Bruce Porter, editors. Handbook of Knowledge Representation. Elsevier, 2008.

17. Hirofumi Katsuno and Alberto O. Mendelzon. Propositional knowledge base revision and minimal change. AIJ, 52(3):263-294, 1992.

18. Jooyhung Lee and Vladimir Lifschitz. Loop formulas for disjunctive logic programs. In ICLP-2003, V. 2916 of LNCS, 451-465, Mumbai, India, 2003. Springer.

19. Vladimir Lifschitz, David Pearce, and Agustín Valverde. Strongly equivalent logic programs. TOCL, 2(4):526-541, 2001.

20. Fangzhen Lin and Yuting Zhao. ASS AT: computing answer sets of a logic program by SAT solvers. AIJ, 157(1-2):115-137, 2004.

21. David Pearce, Hans Tompits, and Stefan Woltran. Characterising equilibrium logic and nested logic programs: Reductions and complexity. TPLP, 9(5):565-616, 2009.

22. Wei Liu and Mary-Anne Williams. A framework for multi-agent belief revision. Studia Logica, 67(2):291-312, 2001.

23. Pavlos Peppas. Handbook of Knowledge Representation, chapter ch. 8 Belief Revision, 317360. Elsevier, 2008.

24. Chiaki Sakama and Katsumi Inoue. An abductive framework for computing knowledge base updates. TPLP, 3(6):671-713, 2003.

25. Ken Satoh. Nonmonotonic reasoning by minimal belief revision. In FGCS-1988, 455-462, Tokyo, Japan, 1988.

26. Luciano Serafini and Paolo Bouquet. Comparing formal theories of context in AI. AIJ, 155(1-2):41-67, 2004.

27. Yan Zhang and Norman Y. Foo. Towards generalized rule-based updates. In IJCAI-1997, 82-88. Morgan Kaufmann, 1997. 